\title{
Effect of Topical Emollient Treatment of Preterm Neonates in Bangladesh on Invasion of Pathogens Into the Bloodstream
}

\author{
GARY L. DARMSTADT, SAMIR K. SAHA, A.S.M. NAWSHAD UDDIN AHMED, YOONJOUNG CHOI, \\ M.A.K. AZAD CHOWDHURY, MAKSUDA ISLAM, PAUL A. LAW, AND SAIFUDDIN AHMED
}

\begin{abstract}
Departments of International Health [G.L.D., Y.C.] and Population and Family Health Sciences [S.A.], Bloomberg School of Public Health, Johns Hopkins University, Baltimore, Maryland 21205; Departments of Microbiology [S.K.S., M.I.] and Neonatology [A.S.M.N.U.A., M.A.K.A.C.], Bangladesh Institute of Child Health, Dhaka Shishu Hospital, Dhaka 1207, Bangladesh; Department of Pediatrics [A.S.M.N.U.A], Kumudini Women's Medical College, Postal Code 1940, Mirzapur, Tangail, Bangladesh; Department of Informatics [P.A.L.], Kennedy Krieger Institute, Johns Hopkins Medical Institutions, Baltimore, Maryland 21211
\end{abstract}

\begin{abstract}
Topical emollient therapy may reduce the incidence of serious infections and mortality of preterm infants in developing countries. We tested whether emollient therapy reduced the burden of pathogens on skin and/or prevented bacterial translocation. Neonates $<33$ wk gestational age were randomized to treatment with sunflower seed oil (SSO) or Aquaphor or the untreated control group. Skin condition score and skin cultures were obtained at enrollment and on d 3, 7, and weekly thereafter, and blood cultures were obtained for episodes of suspected nosocomial sepsis. For analysis, blood cultures were paired with skin cultures obtained $0-3 \mathrm{~d}$ before the blood culture. Skin condition scores at $3 \mathrm{~d}$ were better in patients treated with either emollient compared with untreated controls; however, skin flora was similar across the groups. The SSO group showed a $72 \%$ elevated odds of having a false-positive (FP) skin culture associated with a negative blood culture (i.e. skin flora blocked from entry into blood) compared with the control group. Topical therapy with SSO reduced the passage of pathogens from the skin surface into the bloodstream of preterm infants. (Pediatr Res 61: 588-593, 2007)
\end{abstract}

$\mathrm{R}^{\mathrm{e}}$ ecent evidence suggests that health and development of the skin barrier is a critical determinant in risk of serious infections and mortality in preterm low birth weight (LBW) infants in developing countries (1). In a community-based trial in Nepal, a single cleansing of the skin of LBW newborns using dilute chlorhexidine antiseptic solution as soon as possible after birth reduced mortality by $28 \%$ (2). Application of oils to the skin of newborn infants is nearly a universal domiciliary practice in South Asia $(3,4)$. This practice has evolved empirically, however, and recent evidence suggests that the most commonly used oil (mustard oil) during neonatal massage may be toxic to the skin barrier, whereas use of alternative skin barrier-enhancing products may be of sub-

Received September 25, 2006; accepted December 13, 2006.

Correspondence: Gary L. Darmstadt, M.D., Johns Hopkins University, Bloomberg School of Public Health, Department of International Health E8153, 615 North Wolfe Street, Baltimore, MD 21205; e-mail: gdarmsta@jhsph.edu

This study was supported by the Thrasher Research Fund; the Office of Health, Infectious Diseases and Nutrition, Global Health Bureau, United States Agency for International Development (USAID) (award HRN-A-00-96-90006-00); Save the Children/USA through a grant from the Bill \& Melinda Gates Foundation; and the Society for Pediatric Dermatology.

DOI: $10.1203 /$ pdr.0b013e3180459f75 stantial public health benefit (5). In Egypt (6) and Bangladesh (7), topical applications of emollients to enhance skin barrier function in preterm infants reduced the incidence of nosocomial, culture-proven sepsis by about $40 \%-55 \%$, and also reduced mortality in the trial in Bangladesh (GL Darmstadt, unpublished data).

Although topical emollient therapy has been shown to have a number of potential benefits that stem from improved skin barrier function (8-10), widespread adoption of strategies to improve skin barrier function as a means to improve newborn health and survival has not yet occurred. Further information is needed on the impact of topical emollient therapy in a variety of developing country hospital and community settings and on the mechanism for reduction of nosocomial infections. The skin is recognized as an important potential portal for invasion of pathogens into the bloodstream, based on data from laboratory and animal experiments as well as observations of human diseases such as invasive streptococcal skin infections $(2,11,12)$. To date, however, few clinical data are available on the mechanism for the modulating effect of skin barrier-enhancing products on entry of skin pathogens into the bloodstream to cause sepsis. Previous research has suggested, however, that emollient therapy does not quantitatively or qualitatively change the skin flora $(13,14)$, raising the untested hypothesis that passage of pathogens residing on the skin into the blood may be impeded.

A trial in Bangladesh to assess the impact of topical emollient therapy on incidence of sepsis and mortality provided the opportunity to test the following potential mechanisms for reduction in risk of sepsis: (1) reduction in the presence of pathogens on the skin (i.e. an antibacterial effect on the skin flora) and (2) increased skin barrier function, resulting in prevention of bacterial translocation through the skin.

\section{METHODS}

Patients. This study was nested in a prospective, randomized, controlled trial of the impact of topical emollient therapy on health and survival of preterm infants was conducted from December 1998 to July 2003 in the Special Care Nursery of Dhaka Shishu Hospital, the largest tertiary care

Abbreviations: FN, false negative; FP, false positive; SSO, sunflower seed oil; $\mathbf{T P}$, true positive 
children's hospital in Bangladesh. The hospital has no delivery facility, and all neonates were out-born. Preterm (gestational age $<33$ wk) neonates $\leq 72$ $\mathrm{h}$ old at the time of admission were eligible for the study. Gestational age was determined by maternal dates and the Ballard et al. (15) and the Dubowitz et al. (16) criteria and averaged to determine eligibility. We excluded infants with (1) major congenital anomalies or hydrops fetalis, (2) generalized skin disease likely to produce a defect in epidermal barrier function, (3) a structural defect of the skin involving $>5 \%$ body surface area, (4) conditions requiring a major surgical procedure attended by a high rate of infectious complications, (5) clinically evident skin infection, and (6) those whom the admitting physician, based on clinical judgment, thought would die within $48 \mathrm{~h}$. Background information on demographic and clinical characteristics of patients and their mothers was obtained, including maternal obstetric history, birth history, and patient medical history before enrollment.

A data safety and monitoring board reviewed study progress once or twice yearly. Oral consent from parents or guardian was required before enrollment. The study protocol was approved by the Johns Hopkins Committee for Human Research and the Ethical Review Committee of Dhaka Shishu Hospital and was registered at www.clinicaltrials.gov, \#98-04-21-03-2.

Study design. Eligible neonates were allocated to strata based on gestational age $[(1)<30 \mathrm{wk}$ or $(2) \geq 30 \mathrm{wk}]$ and randomly assigned by a study nurse to one of two treatment groups [high-linoleate SSO (Omega Nutrition, Bellingham, WA) or Aquaphor Original Emollient Ointment (Beiersdorf, Norwalk, CT)] or the untreated control group. Physicians were unaware of the randomization results. Detailed information on randomization and masking among physicians was described previously (6).

Emollient treatment. All neonates received an initial bath in lukewarm water to remove potential exogenous substances from the skin. Emollient was applied by nurses to the entire body surface, except for the scalp and face or intravascular catheter sites of neonates assigned to the treatment arms three times daily for the first $14 \mathrm{~d}$ and then twice daily until discharge. A dosing schedule ( $4 \mathrm{~g}$ of emollient per $\mathrm{kg}$ body weight per treatment) was used, and nurses were trained and supervised in proper application techniques to minimize the risk of skin injury and the potential for spread of fecal flora, as described previously (6). To reduce the potential for contamination and oxidative breakdown of emollients, we refrigerated them, replaced the stock with fresh products every 2 mo, prepared fresh containers of SSO every 2-3 $\mathrm{d}$ for use at the bedside of individual patients, enforced adherence to sterile procedures during dispensing and application, and evaluated weekly cultures of individual patient SSO samples for contamination. Aquaphor was supplied by Beiersdorf in small tubes that contained enough ointment for just one to two applications, obviating risk of contamination.

Neonates in the control group received standard skin care for the Special Care Nursery, which did not include application of topical emollients or other measures to prevent skin breakdown or modify skin barrier function. Neonates in the treatment and control groups otherwise received the same general care, as described previously (6). Patients were kept in an open crib or nonhumidified isolette. Indwelling intravascular catheters, oxygen therapy, and gavage feeding were available in the nursery; however, no patients received mechanical ventilation or parenteral alimentation.

Outcome assessment. Skin condition, as a gross measure of barrier integrity, was assessed at the time of enrollment and on d 3, 7, 14, 21, and 28, as long as the infant remained in the hospital, based on a 9-point scale scoring system (7).

Baseline blood cultures were obtained for all neonates within $48 \mathrm{~h}$ of enrollment to identify preexisting infection before initiating emollient or antibiotic treatment. A subsequent blood culture was obtained if sepsis was suspected clinically. Further information on clinical indications for blood cultures, culture methods, and quality control measures and the algorithm used to identify nosocomial infections were described in detail elsewhere (6). Bacterial pathogens were identified in blood cultures using standard techniques (17). Any culture with multiple organisms was considered contaminated and was excluded from the study.

To assess the cutaneous flora, skin swabs were obtained from three body sites (right axilla, supraumbilical, and right inguinal regions) of each neonate and processed by a modified swab-wash method $(13,18)$. Skin swab specimens were collected from a $4-\mathrm{cm}^{2}$ area of skin, delineated by a sterile template, at the indicated site using a sterile swab by rubbing gently five times horizontally and twice vertically within the sampling area. Baseline skin cultures were obtained from all neonates at enrollment, before any treatment. Follow-up cultures were taken routinely on d 3, 7, 14, and 21 of hospitalization. Follow-up cultures were obtained $8 \mathrm{~h}$ after the previous application of emollient.

Swabs of the skin were placed immediately in $1.0 \mathrm{~mL}$ of phosphatebuffered saline ( $\mathrm{pH}$ 7.0) and transported within $30 \mathrm{~min}$ to the microbiology laboratory. The swab in buffer was vortexed, and 10 -fold serial dilutions were made. Sheep blood and MacConkey agar plates were inoculated with $10 \mu \mathrm{L}$ of undiluted solution and three consecutive 10-fold dilutions. Plates were incubated at $37^{\circ} \mathrm{C}$, and identification and sensitivity testing of isolates was done after $48 \mathrm{~h}$ following standard procedures (17). If one or more pathogens was isolated, the skin culture specimen was considered positive.

Analysis. To study the association between blood and skin cultures, we generated skin-blood comparison pairs from 819 blood and 4371 skin cultures. Blood cultures were considered to be index events, and each skin culture obtained 0-3 d before the blood culture was compared with the blood culture because a 3-d incubation time is suggested to be enough to detect clinical infections (19). An algorithm, adapted from Evans et al. (20), was used to categorize the relationship of each pair into one of five possibilities as shown in Figure 1. When multiple organisms were isolated from a skin culture and a blood culture was positive, we classified the comparison as true positive (TP) if any of the skin pathogens matched with the blood pathogen, and as FP if none of the skin pathogens matched with the blood pathogen.

The main specific aim of the study was to explore treatment-differential entry of skin pathogens into blood, and each classification implied different treatment effects on systemic infection (Fig. 1). A TP (i.e. the same organism on the skin and in the blood) implied that the source of systemic infection may have been pathogens on the skin, although the implication cannot be verified definitively based on the data presented here, lacking molecular markers. We examined odds ratios (ORs) of being TP by treatment group among positive blood-skin pairs and hypothesized that the OR would be $<1$ if treatment reduced skin pathogen entry into blood, compared with a no intervention. An FP (i.e. positive skin culture) with a negative blood culture suggested that a pathogen on skin did not enter into the blood, potentially due to improved skin barrier function. We therefore estimated ORs of being FP with a negative blood culture among positive skin pairs, with the hypothesis that the OR would be $>1$ if treatment prevented skin pathogen entry into the blood compared with no intervention. Finally, FP with a positive blood culture (i.e. the pathogen on the skin and in the blood did not match) indicated that since emollient treatment was not expected to alter skin flora $(13,14)$ and the blood pathogen was not found on the skin, the blood isolate most likely came from a source other than skin, such as the gastrointestinal tract or lungs, despite the presence of another type of surface pathogen. We hypothesized that this mechanism of infection may be altered by topical treatment if the treatment has distal effects to prevent a pathogen's entry into the blood through sources other than the skin. Thus, we additionally estimated ORs among positive skin pairs of being FP with a positive blood culture.

We stratified skin-blood comparison pairs by timing of the blood culture into (1) baseline and (2) nosocomial pairs. Baseline pairs were those in which both the blood and skin cultures were taken within $48 \mathrm{~h}$ of enrollment, before any topical emollient or antibiotic treatment intervention. Nosocomial pairs were those in which a blood culture was obtained after the first $4 \mathrm{~d}$ of hospitalization and the blood culture met the definition of nosocomial infection as described previously (6). All other pairs, involving either a blood culture taken after the baseline but before d 5 of hospitalization or a blood

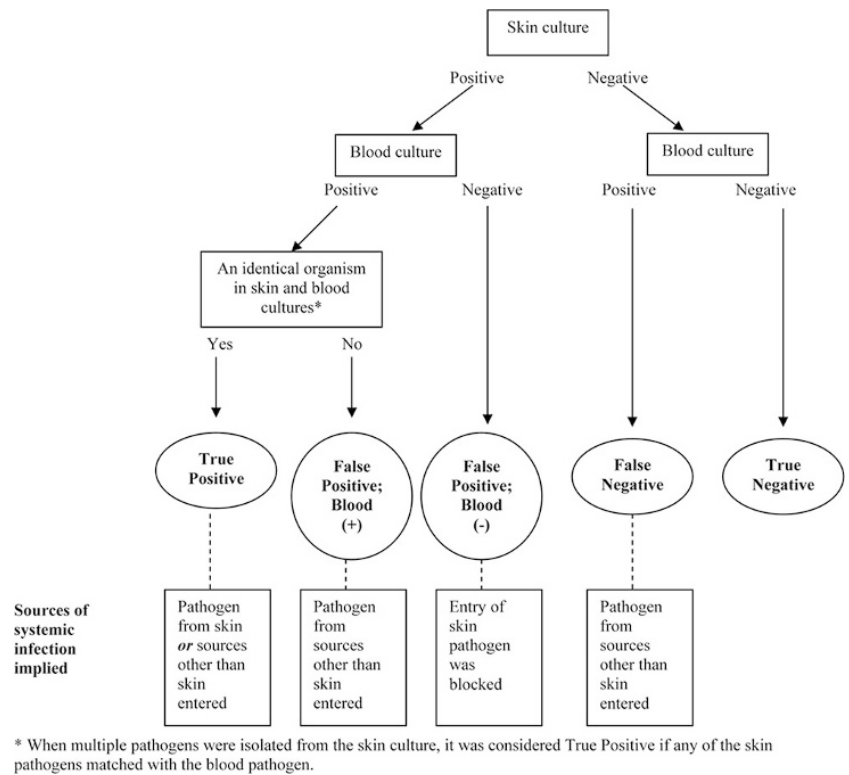

Figure 1. Algorithm to classify skin cultures, with reference to subsequently obtained blood cultures. 
culture taken on d 5 or later but that did not meet the nosocomial infection definition, were excluded from the analysis.

Data were double-entered in Epi Info version 6 (Centers for Disease Control and Prevention, Atlanta, GA), and analyses were conducted using STATA 8.0 statistical software (Stata Corporation, College Station, TX). We analyzed data using the generalized estimating equation model with binomial link, adjusted for weight at admission. We also used robust variance estimation methods to address intracluster correlation in the model. A $p$ value of 0.05 was considered statistically significant.

\section{RESULTS}

Patients. The trial profile of the patients reported in this nested study was presented previously (6). Demographic and clinical characteristics of the study patients were similar across groups, also as shown previously.

Skin condition. Mean skin score gradually increased in all groups; however, patients treated with SSO or Aquaphor had lower skin scores (i.e. better skin condition) both at the baseline and at most follow-up examinations, compared with the control group (Table 1). The SSO and Aquaphor groups also showed smaller incremental increases in skin scores between the baseline and the d-3 follow-up, compared with the control group; however, there was no group differential in skin score changes thereafter.

Skin colonization. Patients in the Aquaphor group had lower baseline skin culture positivity rates, but baseline rates in the SSO group were similar compared with the control group (Table 2). There was no group difference in positive skin culture rates at any follow-up time. Pathogen distribution among positive skin cultures was comparable across groups, both at baseline and all follow-up times. A total of nine bacterial species were isolated from skin cultures. Among the positive skin cultures, there was no group difference in mean colony counts per $1 \mathrm{~cm}^{2}$ of surface area at baseline or at any follow-up time, but all three groups showed a highly skewed distribution to the right (i.e. with outliers showing significantly high colony count values) (results not shown).

Blood-skin pathogen pairs. Blood culture positivity rates were lower among the baseline cultures ( $n=496,5.6 \%)$ compared with the follow-up cultures $(n=323,50.5 \%)$, but comparable by group: $3.8 \%$ in SSO $(n=159), 7.1 \%$ in Aquaphor $(n=156)$, and $6.1 \%$ in the control $(n=181)$ group.
A total of 2070 skin-blood comparison pairs were analyzed, 1146 baseline pairs and 624 nosocomial pairs (Table 3). The association distributions differed markedly between baseline and nosocomial pairs. Among the baseline and nosocomial pairs, $51 \%$ and $63 \%$, respectively, had positive skin cultures (i.e. were $\mathrm{TP}$ or $\mathrm{FP}$ ), and $6 \%$ and $50 \%$, respectively, had positive blood cultures [i.e. TP, FP with a positive blood culture, or false negative (FN)]. Among the positive-skin pairs, only $2 \%$ and $7 \%$ were $\mathrm{TP}$ from the baseline and nosocomial pairs, respectively. Furthermore, among the nosocomial pairs, overall only $9 \%$ of positive-blood pairs were TP. The distribution also varied by group among the nosocomial pairs. In particular, among the positive-skin pairs, around $5 \%$ were TP in both treatment groups compared with $11 \%$ in the control group. Distributions were similar for nosocomial pairs analyzed based on an interval of $0-6 \mathrm{~d}$ compared with 0-3 d (data not shown).

Blood-skin pairs positive for skin colonization were further analyzed for estimated, adjusted ORs of three outcome measures by group (Table 4). Among the baseline comparisons, before any treatment, there were no significant group differentials in ORs for any outcome measure.

Among the nosocomial comparisons, however, the SSO group showed a $72 \%$ elevated odds of FP with a negative blood culture and a $38 \%$ reduced odds of FP with a positive blood culture compared with the control group (Table 4). In addition, neonates $<1250 \mathrm{~g}(n=225)$ had a higher odds of being FP with a negative blood culture $(\mathrm{OR}=1.63,95 \% \mathrm{CI}$ : 1.06-2.50) and a lower odds of being FP with a positive blood culture (OR $=0.66,95 \% \mathrm{CI}: 0.44-0.99)$ compared with those $\geq 1250 \mathrm{~g}(n=270)$, controlled for group allocation. Interaction between group effects and weight was not significant (results not shown). We adjusted for skin culture sites, antibiotic use before admission, respiratory distress at enrollment, and number of antibiotic treatment days during hospitalization, but there was no meaningful difference in results (results not shown).

We further analyzed skin and blood pathogen distributions by classification among the nosocomial pairs. Distributions of skin pathogens were similar between the two types of FP pairs

Table 1. Skin condition by group

\begin{tabular}{|c|c|c|c|c|c|c|}
\hline & \multicolumn{2}{|c|}{ Control } & \multicolumn{2}{|c|}{ Aquaphor } & \multicolumn{2}{|c|}{ Sunflower seed oil } \\
\hline & $n$ & Mean (SD) & $n$ & Mean (SD) & $n$ & Mean (SD) \\
\hline \multicolumn{7}{|l|}{ Skin score } \\
\hline Baseline & 178 & $0.13(048)$ & 157 & $0.04(0.19)^{*}$ & 156 & $0.03(0.16) \dagger$ \\
\hline $3 \mathrm{~d}$ & 149 & $0.46(0.72)$ & 135 & $0.13(0.36) \dagger$ & 132 & $0.19(0.55) \dagger$ \\
\hline $7 \mathrm{~d}$ & 107 & $1.13(0.70)$ & 97 & $0.70(0.69) \dagger$ & 86 & $0.77(0.48) \dagger$ \\
\hline $14 \mathrm{~d}$ & 68 & $1.74(0.87)$ & 59 & $1.10(0.40) \dagger$ & 47 & $1.23(0.52) \dagger$ \\
\hline $21 \mathrm{~d}$ & 31 & $2.00(0.73)$ & 30 & $1.53(0.57) \dagger$ & 29 & $1.93(0.46)$ \\
\hline $28 \mathrm{~d}$ & 9 & $2.22(0.97)$ & 9 & $2.11(0.33)$ & 8 & $2.13(0.35)$ \\
\hline \multicolumn{7}{|l|}{ Skin score change } \\
\hline Baseline to $3 \mathrm{~d}$ & 149 & $0.34(0.52)$ & 135 & $0.09(0.29) \dagger$ & 132 & $0.16(0.54) \dagger$ \\
\hline $3-7 d$ & 107 & $0.59(0.49)$ & 97 & $0.57(0.68)$ & 86 & $0.65(0.50)$ \\
\hline $7-14 \mathrm{~d}$ & 68 & $0.59(0.55)$ & 59 & $0.46(0.50)$ & 47 & $0.47(0.58)$ \\
\hline $14-21 \mathrm{~d}$ & 31 & $0.52(0.63)$ & 30 & $0.43(0.57)$ & 29 & $0.59(0.50)$ \\
\hline $21-28 \mathrm{~d}$ & 9 & $0.10(0.10)$ & 9 & $0.10(0.10)$ & 8 & $0.04(0.07)$ \\
\hline
\end{tabular}

$* p<0.05$ based on $t$ test $/ \chi^{2}$ test for differences compared to the control group.

$\dagger p<0.01$ based on $t$ test $/ \chi^{2}$ test for differences compared with the control group. 
Table 2. Skin colonization by site, timing, and group

\begin{tabular}{|c|c|c|c|c|c|c|c|c|c|c|}
\hline \multirow[b]{3}{*}{ Site } & \multirow[b]{3}{*}{ Timing } & \multicolumn{3}{|c|}{ Control } & \multicolumn{3}{|c|}{ Aquaphor } & \multicolumn{3}{|c|}{ SSO } \\
\hline & & \multicolumn{2}{|r|}{$n$} & \multirow{2}{*}{$\begin{array}{c}\% \\
\text { Average } \\
\text { positive rate }\end{array}$} & \multicolumn{2}{|r|}{$n$} & \multirow{2}{*}{$\begin{array}{c}\% \\
\text { Average } \\
\text { positive rate }\end{array}$} & \multicolumn{2}{|r|}{$n$} & \multirow{2}{*}{$\begin{array}{c}\% \\
\text { Average } \\
\text { positive rate }\end{array}$} \\
\hline & & Total & Positive & & Total & Positive & & Total & Positive & \\
\hline \multicolumn{11}{|l|}{ Axilla } \\
\hline & Baseline & 181 & 109 & $(60.2)$ & 157 & 74 & $(47.1)^{*}$ & 159 & 97 & (61.0) \\
\hline & $3 \mathrm{~d}$ & 150 & 97 & (64.7) & 140 & 78 & (55.7) & 141 & 98 & (69.5) \\
\hline & $7 \mathrm{~d}$ & 106 & 76 & (71.7) & 93 & 62 & (66.7) & 88 & 56 & (63.6) \\
\hline & $14 \mathrm{~d}$ & 63 & 42 & (66.7) & 58 & 38 & $(65.5)$ & 44 & 30 & (68.2) \\
\hline & $21 \mathrm{~d}$ & 25 & 13 & $(52.0)$ & 25 & 17 & $(68.0)$ & 27 & 20 & (74.1) \\
\hline \multicolumn{11}{|l|}{ Umbilical } \\
\hline & Baseline & 181 & 79 & (43.6) & 157 & 48 & $(30.6)^{*}$ & 159 & 67 & $(42.1)$ \\
\hline & $3 \mathrm{~d}$ & 150 & 63 & $(42.0)$ & 140 & 65 & $(46.4)$ & 141 & 67 & (47.5) \\
\hline & $7 \mathrm{~d}$ & 106 & 47 & (44.3) & 93 & 45 & (48.4) & 88 & 50 & (56.8) \\
\hline & $14 \mathrm{~d}$ & 63 & 30 & (47.6) & 58 & 30 & (51.7) & 44 & 24 & $(54.5)$ \\
\hline & $21 \mathrm{~d}$ & 25 & 11 & $(44.0)$ & 25 & 14 & $(56.0)$ & 27 & 16 & $(59.3)$ \\
\hline \multicolumn{11}{|l|}{ Inguinal } \\
\hline & Baseline & 181 & 106 & (58.6) & 157 & 80 & $(51.0)$ & 159 & 94 & (59.1) \\
\hline & $3 \mathrm{~d}$ & 150 & 93 & $(62.0)$ & 140 & 78 & $(55.7)$ & 141 & 93 & $(66.0)$ \\
\hline & $7 \mathrm{~d}$ & 106 & 70 & $(66.0)$ & 93 & 60 & $(64.5)$ & 88 & 55 & (62.5) \\
\hline & $14 \mathrm{~d}$ & 63 & 38 & $(60.3)$ & 58 & 41 & $(70.7)$ & 44 & 29 & $(65.9)$ \\
\hline & $21 \mathrm{~d}$ & 25 & 16 & $(64.0)$ & 25 & 15 & $(60.0)$ & 27 & 23 & $(85.2)$ \\
\hline \multicolumn{11}{|c|}{ Pathogen distribution $(\%) \dagger$} \\
\hline & Klebsiella pneumoniae & & 63 & $(17.7)$ & & 42 & (17.4) & & 53 & $(16.6)$ \\
\hline & Staphylococcus aureus & & 78 & $(21.9)$ & & 51 & $(21.1)$ & & 78 & $(24.5)$ \\
\hline & Acinetobacter spp. & & 96 & $(27.0)$ & & 65 & $(26.9)$ & & 82 & $(25.7)$ \\
\hline & Pseudomonas aeruginosa & & 32 & $(9.0)$ & & 19 & $(7.9)$ & & 26 & $(8.2)$ \\
\hline & CNS $\ddagger$ & & 54 & $(15.2)$ & & 50 & $(20.7)$ & & 61 & $(19.1)$ \\
\hline & Others & & 33 & $(9.3)$ & & 15 & $(6.2)$ & & 19 & $(6.0)$ \\
\hline & Total & & 356 & $(100.0)$ & & 242 & $(100.0)$ & & 319 & $(100.0)$ \\
\hline
\end{tabular}

$* p<0.05$ based on $t$ test $/ \chi^{2}$ test for differences compared with the control group.

$\dagger$ Overall distribution of pathogens among positive skin cultures across sites and timing of cultures.

$\ddagger$ CNS refers to coagulase-negative Staphylococcus.

§ Others include Enterobacter spp., Salmonella spp., Candida spp., and Escherichia coli.

Table 3. Distribution of comparison pairs by timing of blood culture and treatment group

\begin{tabular}{|c|c|c|c|c|c|}
\hline \multirow[b]{2}{*}{$\begin{array}{l}\text { Blood-skin culture comparison } \\
\text { pairs (see Fig. 1) }\end{array}$} & \multirow[b]{2}{*}{$\begin{array}{c}\text { Baseline pairs, } \\
\text { total }(n=1446) \\
n(\%)\end{array}$} & \multirow[b]{2}{*}{$\begin{array}{c}\text { Nosocomial pairs, } \\
\text { total }^{*}(n=624) \\
n(\%)\end{array}$} & \multicolumn{3}{|c|}{$\begin{array}{l}\text { Treatment group } \\
\text { (among nosocomial pairs) }\end{array}$} \\
\hline & & & $\begin{array}{c}\text { Control }(n=213) \\
n(\%)\end{array}$ & $\begin{array}{c}\text { Aquaphor } \dagger(n=159) \\
n(\%)\end{array}$ & $\begin{array}{c}\mathrm{SSO}+(n=252) \\
n(\%)\end{array}$ \\
\hline $\mathrm{TP}$ & $11(0.8)$ & $28(4.5)$ & $15(7.0)$ & $5(3.1)$ & $8(3.2)$ \\
\hline FP with a positive blood culture & $33(2.3)$ & $172(27.6)$ & $75(35.2)$ & $35(22.0)$ & $62(24.6)$ \\
\hline FP with a negative blood culture & $688(47.6)$ & $191(30.6)$ & $53(24.9)$ & $48(30.2)$ & $90(35.7)$ \\
\hline $\mathrm{FN}$ & $40(2.8)$ & $109(17.5)$ & $42(19.7)$ & $26(16.4)$ & $41(16.3)$ \\
\hline $\mathrm{TN}$ & $674(46.6)$ & 124 (19.9) & $28(13.1)$ & $45(28.3)$ & $51(20.2)$ \\
\hline
\end{tabular}

$* p<0.01\left(\chi^{2}\right.$ test $)$ compared to baseline pairs.

$\dagger p<0.01$ ( $\chi^{2}$ test) compared with the control group.

(results not shown). Distributions of blood pathogens differed by classification (Fig. 2). A total of 11 pathogenic bacterial species were isolated from blood cultures. Although Klebsiella pneumoniae and Acinetobacter spp. comprised the majority of blood pathogens among TPs (82\%), Enterobacter spp., Candida spp., Pseudomonas spp., and Salmonella spp. together accounted for about $59 \%$ and $43 \%$ of total pathogens among FP and FN pairs, respectively.

\section{DISCUSSION}

We have shown previously that emollient therapy reduces the incidence of nosocomial sepsis in preterm infants $(6,7)$.
This study was designed to provide insight into the mechanism for this effect and suggest that topical applications of SSO reduced the entry of pathogens from skin into the bloodstream of preterm infants in Bangladesh. This was demonstrated by analysis showing that infants treated with SSO, compared with the untreated control infants, had a higher odds that a given pathogen was found only on the skin surface and not in the blood (i.e. increased odds of FP with a negative blood culture), suggesting that skin pathogens were more likely to be confined to the skin and failed to gain entry into the bloodstream. The relationship between skin-blood pairs at baseline did not differ by treatment group, indicating that the 
Table 4. Adjusted ORs of preventing skin-to-blood entry of a pathogen among positive skin culture comparison pairs

\begin{tabular}{llc}
\hline \multicolumn{1}{c}{ Treatment group } & Adjusted OR* $(95 \%$ CI $)$ & $p$ \\
\hline $\begin{array}{l}\text { Baseline }(n=728) \\
\text { TP }\end{array}$ & $1.55(0.75-3.20)$ & \\
$\quad$ Aquaphor & $0.83(0.34-2.04)$ & 0.239 \\
$\quad$ SSO & $0.863(0.53-1.39)$ & 0.531 \\
FP with a negative blood culture & $1.39(0.81-2.39)$ & 0.236 \\
$\quad$ Aquaphor & & \\
$\quad$ SSO & $0.99(0.59-1.67)$ & 0.977 \\
FP with a positive blood culture & $0.71(0.29-1.27)$ & 0.246 \\
$\quad$ Aquaphor & & \\
$\quad$ SSO & $0.84(0.41-1.71)$ & 0.636 \\
Nosocomial ( $n=391)$ & $0.82(0.45-1.50)$ & 0.511 \\
TP $\quad$ & $1.50(0.87-2.60)$ & 0.149 \\
$\quad$ Aquaphor & $1.72(1.06-2.81)$ & 0.030 \\
$\quad$ SSO & & \\
FP with a negative blood culture & & \\
$\quad$ Aquaphor & $0.69(0.41-1.17)$ & 0.169 \\
$\quad$ SSO & $0.62(0.39-0.98)$ & 0.039 \\
FP with a positive blood culture &
\end{tabular}

*Adjusted for weight at admission $(<1250 \mathrm{~g} v s \geq 1250 \mathrm{~g})$.

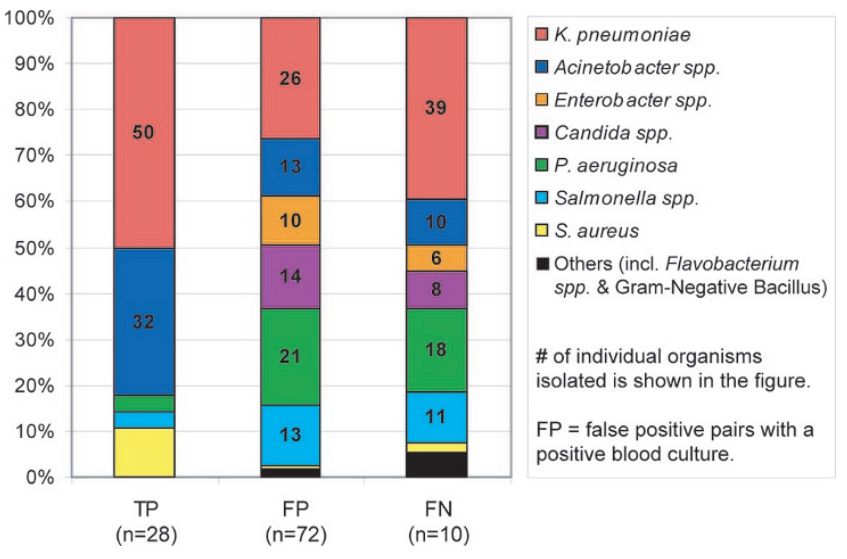

Figure 2. Distribution of blood pathogens by classification.

groups were balanced at enrollment but diverged due to the treatment effect. Consistent with the finding reported previously that treatment with Aquaphor had less impact than SSO on reducing the incidence of nosocomial infections (6), in this study, we found that Aquaphor treatment had limited effect on the relationships between skin and blood pathogens (Table 3). In addition, we did not see significant differentials in odds of finding a given skin pathogen in the blood (i.e. odds of TP) for any treatment group.

Although treatment with SSO appeared to reduce the entry of skin pathogens into blood, our data indicate that only $10 \%$ of cases of nosocomial sepsis may have originated from skin (i.e. one in 10 positive blood nosocomial pairs was TP). The reduced odds of FP with a positive blood culture suggests that treatment with SSO may also prevent sepsis originating from sites other than the skin. This is further supported by the observation that SSO treatment reduced the incidence of nosocomial infections in clinical trials by about half $(6,7)$, which could only come about if mechanisms other than prevention of skin-derived sepsis were operating, given our data suggesting that $10 \%$ of cases of sepsis may be skin derived. This effect was not seen with Aquaphor, as expected for a product that is thought to largely affect mechanical barrier function (21-23) and to have little potential for systemic absorption or distal effects. Another possible explanation for this result is a failure in these instances to detect the pathogen on the skin while isolating it from blood, when in fact the blood pathogen came from the skin (i.e. it was TP). This seems unlikely, however, because we know that the skin flora was unaffected by emollient treatment, and thus one would not expect a differential failure of isolation of skin flora in the SSO arm relative to the control.

The mechanism for this protective effect of SSO at nonskin sites is unknown, but absorption of topically applied fatty acids through skin into the bloodstream, which has been demonstrated in animal models and humans, including preterm infants $(8,24,25)$, could potentially modulate barrier function and other aspects of immune function at other sites for invasion of pathogens, such as the gastrointestinal or pulmonary mucosae. Essential fatty acid deficiency has been associated with increased risk of translocation of intestinal bacteria (26), whereas various diets containing essential fatty acids have been shown to modulate inflammation, improve gut barrier function, reduce gutderived sepsis, improve pulmonary function, and reduce mortality (27-29).

Skin condition scores, a gross indication of skin barrier health, were better in both treatment groups in the first few days after starting topical therapy, suggesting that improved skin barrier function may play a role, particularly during the first few days after starting therapy. Others have reported improved skin condition $(7,13)$ and reduced rates of transepidermal water loss, a more precise surrogate measure of skin barrier function $(13,14,30)$, with emollient treatment. However, we were unable to measure transepidermal water loss in the study setting, and thus we lack direct, definitive data on skin barrier function in this study. Nevertheless, we were able to rule out changes in skin flora as the reason for the results that we observed. Moreover, our tests for antibacterial properties of SSO and Aquaphor have indicated that growth in the laboratory is unaffected.

Analysis of blood pathogens according to their relationship to skin isolates (Fig. 2) suggests that blood pathogens that could not have come from skin (i.e. FP with a positive blood culture or $\mathrm{FN}$ ) were a mix of various pathogens typically associated with gut-derived sepsis. Of note, the pattern of blood isolates with a potential skin source (i.e. TP) differed markedly and was weighted toward Klebsiella or Acinetobacter spp., suggesting that these organisms have a relatively greater propensity for entry through the skin. In contrast, several common pathogens in neonatal species were rare to nonexistent among the TPs (i.e. Enterobacter spp, Pseudomonas aeruginosa, Candida spp., Salmonella spp.), suggesting that these organisms gain entry primarily through nonskin sites. In addition, distributions of skin pathogens were comparable between FP with a positive and FP with a negative blood culture, implying that infection through sources other than skin was independent of skin pathogen type. 
Our matching for skin-blood isolates was done only on the basis of organism identification; future research using molecular methods could demonstrate conclusively whether skin-blood matching pairs (e.g. TPs) were composed of the identical isolate, providing further evidence that a given organism found in the blood could have originated from the skin. A limited number of putative virulence factors associated with clinical syndromes caused by $K$. pneumoniae and Acinetobacter spp. have been identified $(31,32)$; however, further research is needed to define factors associated with their invasiveness through the skin barrier to cause sepsis.

In conclusion, the skin is a potentially important portal of entry for pathogens that cause sepsis in preterm infants in Bangladesh, particularly Klebsiella and Acinetobacter, the two main pathogens in nosocomial neonatal sepsis in this setting. We furthermore demonstrate that topical therapy with SSO modulated this relationship and prevented sepsis due to organisms colonizing the skin. However, topical therapy also may have reduced the risk of sepsis via other portals, such as the gastrointestinal tract, although this is unproven. Further research is needed to elucidate the relationship between skin therapy and modulation of infection through both skin and nonskin portals of entry. Meanwhile, skin barrier therapy is a promising strategy for reducing sepsis among preterm hospitalized newborns, and further research is also needed to evaluate the impact of this intervention in other settings, such as in developing country communities where the majority of global neonatal deaths due to sepsis occur.

Acknowledgments. SSO was kindly provided by Omega Nutrition Inc., Bellingham, WA, and Aquaphor Original Emollient Ointment was donated by Beiersdorf Inc., Norwalk, CT. The authors thank Kim Mulholland, Barbara Stoll, and William Blackwelder for their guidance as members of the study's Data and Safety Monitoring Board.

\section{REFERENCES}

1. Bhutta ZA, Darmstadt GL, Hasan B, Haws RA 2005 Community-based interventions for improving perinatal and neonatal health outcomes in developing countries: review of the evidence. Pediatrics 115:519-617

2. Tielsch JM, Darmstadt GL, Mullany LC, Khatry SK, Katz J, Leclerq SC, Shrestha S, Adhikari R 2007 Impact of newborn skin-cleansing with chlorhexidine on neonatal mortality in southern Nepal: a community-based, cluster-randomized trial. Pediatrics [Epub ahead of print]

3. Mullany LC, Darmstadt GL, Khatry SK, Tielsch JM 2005 Traditional massage of newborns in Nepal: implications for trials of improved practice. J Trop Pediatr 51:82-86

4. Darmstadt GL, Saha SK 2002 Traditional practice of oil massage of neonates in Bangladesh. J Health Popul Nutr 20:184-188

5. Darmstadt GL, Mao-Qiang M, Chi E, Saha SK, Ziboh VA, Black RE, Santosham M, Elias PM 2002 Impact of topical oils on the skin barrier: possible implications for neonatal health in developing countries. Acta Paediatr 91:546-554
6. Darmstadt GL, Saha SK, Ahmed NU, Chowdhury MA, Law PA, Ahmed S, Alam MA, Black RE, Santosham M 2005 Effect of topical treatment with skin barrierenhancing emollients on nosocomial infections in preterm infants in Bangladesh: a randomized controlled trial. Lancet 365:1039-1045

7. Darmstadt GL, Badrawi N, Law PA, Alam A, Ahmed S, Husein MH, Winch P, Santosham M, Gipson R 2004 Topical therapy with sunflower seed oil prevents nosocomial infections and mortality in premature babies in Egypt: a randomized, controlled clinical trial. Pediatr Infect Dis J 23:719-725

8. Fernandez A, Patkar S, Chawla C, Taskar T, Prabhu S 1987 Oil application in preterm babies, a source of warmth and nutrition. Indian Pediatr 24:1111-1117

9. Agarwal KN, Gupta A, Pushkarna R, Bhargava S, Faridi M, Prabhuy MK 2000 Effect of massage and use of oil on growth, blood flow and sleep pattern in infants. Indian J Med Res 112:212-217

10. Sankaranarayanan K, Mondkar JA, Chauhan MM, Mascarenhas BM, Mainkar AR, Salvi RY 2005 Oil massage in neonates: an open randomized controlled study of coconut versus mineral oil. Indian Pediatr 42:877-884

11. Stevens DL, Tanner MH, Winship J, Swarts R, Ries KM, Schlievert PM, Kaplan E 1989 Severe group A streptococcal infections associated with a toxic shock-like syndrome and scarlet fever toxin. N Engl J Med 321:1-9

12. Darmstadt G, Saha S, Ahmed A, Khatun M, Chowdhury M 2003 The skin as a potential portal of entry for invasive infections in neonates. Perinatol 5:205-212

13. Nopper AJ, Horii KA, Sookdeo-Drost S, Wang TH, Mancini AJ, Lane AT 1996 Topical ointment therapy benefits premature infants. J Pediatr 128:660-669

14. Pabst RC, Starr KP, Qaiyumi S, Schwalbe RS, Gewolb IH 1999 The effect of application of Aquaphor on skin condition, fluid requirements, and bacterial colonization in very low birth weight infants. J Perinatol 19:278-283

15. Ballard JL, Khoury JC, Wedig K, Wang L, Ellers-Walsman BL, Lipp R 1991 New Ballard Score, expanded to include extremely premature infants. J Pediatr 119:417-423

16. Dubowitz LM, Dubowitz V, Goldberg C 1970 Clinical assessment of gestational age in the newborn infant. J Pediatr 77:1-10

17. Cheesebrough M 1987 Medical Laboratory Manual for Tropical Countries, Vol 2. Tropical Health Technology. Butterworth-Heinemann, London, pp 182-183.

18. Keyworth N, Millar MR, Holland KT 1990 Swab-wash method for quantitation of cutaneous microflora. J Clin Microbiol 28:941-943

19. Kumar Y, Qunibi M, Neal TJ, Yoxall CW 2001 Time to positivity of neonatal blood cultures. Arch Dis Child Fetal Neonatal Ed 85:182-186

20. Evans ME, Schaffner W, Federspiel CF, Cotton RB, McKee KT, Stratton CW 1988 Sensitivity, specificity, and predictive value of body surface cultures in a neonatal intensive care unit. JAMA 259:248-252

21. Mao-Qiang M, Brown BE, Wu-Pong S, Feingold KR, Elias PM 1995 Exogenous nonphysiologic vs physiologic lipids. Divergent mechanisms for correction of permeability barrier dysfunction. Arch Dermatol 131:809-816

22. Ghadially R, Halkier-Sorensen L, Elias PM 1992 Effects of petrolatum on stratum corneum structure and function. J Am Acad Dermatol 26:387-396

23. Brown BE, Diembeck W, Hoppe U, Elias PM 1995 Fate of topical hydrocarbons in the skin. J Soc Cosm Chem 46:1-9

24. Friedman Z, Shochat SJ, Maisels J, Marks KH, Lamberth EL Jr 1976 Correction of essential fatty acid deficiency in newborn infants by cutaneous application of sunflower seed oil. Pediatrics 58:650-654.

25. Solanki K, Matnani M, Kale M, Joshi K, Bavdekar A, Bhave S, Pandit A 2005 Transcutaneous absorption of topically massaged oil in neonates. Indian Pediatr 42:9981005

26. Barton RG, Cerra FB, Wells CL 1992 Effect of a diet deficient in essential fatty acids on the translocation of intestinal bacteria. JPEN J Parenter Enteral Nutr 16:122-128

27. Gennari R, Alexander W, Eaves-Pyles T 1995 Effect of different combinations of dietary additives on bacterial translocation and survival in gut-derived sepsis. J Parent Enteral Nutr 19:319-325

28. Pscheidl E, Schywalsky M, Tschaikowsky K, Boke-Prols T 2000 Fish oil-supplemented parenteral diets normalize splanchnic blood flow and improve killing of translocated bacteria in a low-dose endotoxin rate model. Crit Care Med 28:1489-1496

29. Singer P, Theilla M, Fisher H, Gibstein L, Grozovski E, Cohen J 2006 Benefit of an enteral diet enriched with eicosapentaenoic acid and gamma-linolenic acid in ventilated patients with acute lung injury. Crit Care Med 34:1033-1038

30. Grubauer G, Elias PM, Feingold KR 1989 Transepidermal water loss: the signal for recovery of barrier structure and function. J Lipid Res 30:323-333

31. Struve C, Forestier C, Kroqfelt KA 2003 Application of a novel multi-screening signature-tagged mutagenesis assay for identification of Klebsiella pneumoniae genes essential in colonization and infection. Microbiology 149:167-176

32. Joly-Guillou ML 2005 Clinical impact and pathogenicity of Acinetobacter. Clin Microbiol Infect 11:868-873 